\title{
Repeat Computed Tomography Simulation among Patients with Head and Neck Cancer Experiencing Delayed Radiation Therapy during the COVID-19 Pandemic
}

\author{
Benedict Mihangel P. Crisostomo, MD and Lorelei L. Chavez, MD \\ Philippine General Hospital, University of the Philippines Manila
}

\begin{abstract}
Background. Delays in initiation and interruptions in the radiotherapeutic management of head and neck malignancies have radiobiologic implications in allowing for the accelerated repopulation of surviving tumor cells. This phenomenon has been demonstrated to translate into poorer survival outcomes and tumor control, typically manifesting as tumor progression that may require a new radiotherapy set-up. Such adjustments are accounted for with a repeat computed tomography (CT) simulation which entails increased resource costs, patient inconvenience and further treatment delays. Even with maximal efforts to minimize treatment delays, the real-world effects of the current pandemic on the logistics and compliance to daily radiotherapy remain significant. A comparison of the incidence of tumor progression before and during the pandemic is thus investigated, with repeat CT simulation utilized as a surrogate.
\end{abstract}

Objective. This study aims to compare the incidence of repeat CT simulation among patients with head and neck cancer treated before and during the COVID-19 pandemic. Various patient- and/or treatment-related factors, including treatment delay, that may associate with the likelihood of repeat simulation are also investigated in this review.

Methods. Medical and radiation treatment records of patients with head and neck malignancies who underwent CT simulation before and during the pandemic were retrospectively reviewed and prospectively followed up until completion of treatment.

Results. A total of 72 patients were included, with an average age of 45 years (range: $7-82$ ). The median time to radiotherapy initiation was 37 days (SD \pm 37 , range of 0-278) from initial CT simulation and was similar between the groups $(p=0.774)$. Repeat simulation was observed more frequently in the pandemic group $(76.2 \%$ vs $23.8 \%, p=0.007)$. Patients who had delayed radiotherapy initiation $(\mathrm{p}=0.005$, OR $13.59 ; \mathrm{Cl} 2.23-82.87)$ or had treatment interruptions ( $p=0.004$, OR 23.21; $\mathrm{Cl} 2.79-193.29)$ had a higher incidence of repeat simulation.

Conclusion. A higher incidence of repeat simulation was observed in this population treated six months into the pandemic. Delays in radiotherapy initiation and treatment interruptions were significantly associated with repeat simulation. Strategies should be employed to prevent disease progression among this subset of patients to minimize the burden on radiotherapy resources and preserve oncologic outcomes.

Keywords: head and neck cancer, repeat simulation, radiotherapy delay, COVID-19 pandemic

\section{INTRODUCTION}

Corresponding author: Benedict Mihangel P. Crisostomo, MD Philippine General Hospital

University of the Philippines Manila

Taft Avenue, Ermita, Manila 1000, Philippines

Email: bpcrisostomo@up.edu.ph
Cancer of the head and neck region, specifically of the squamous cell histology, afflicts approximately 600,000 patients worldwide, with nearly $60 \%$ having locally advanced but non-metastatic disease. ${ }^{1}$ In the Philippines, 7,497 or $6 \%$ of all new cancer cases diagnosed in the year 2015 alone involved the nasopharynx, oral cavity, larynx, 
and other areas of the pharynx. ${ }^{2}$ Treatment for these neoplasms with such localizations to the head and neck have historically included radiotherapy, chemotherapy, or a combination of both.

Accelerated repopulation is a radiobiologic phenomenon wherein surviving tumor cells or "clonogens" grow rapidly after tumor shrinkage, typically at about 28 days from initiation of radiotherapy. Cytotoxic therapy may trigger this process such that tumor regression paradoxically allows for more rapid proliferation of surviving clonogens. These observations have led to the development of surrogates for anatomic changes in head and neck cancers, using imageguidance during treatment to detect tumor progression. ${ }^{3}$

To prevent recurrence or progression, analyses have been done comparing the overall duration of fractionated radiotherapy in head and neck cancer. For fast-growing tumors with a tendency for accelerated repopulation, treatment is recommended to be completed as soon as practicable and overall treatment time should not be prolonged to assure the efficacy of later fractions. ${ }^{4}$ Higher doses have been observed to be needed in later fractions such that a dose increment of $0.6 \mathrm{~Gy}$ per day is required to compensate for this repopulation. ${ }^{4} \mathrm{~A}$ consensus statement by the American and European Societies for Radiation Oncology made through a rapid Delphi process recommended that treatment for this subsite should not be postponed by more than 4-6 weeks with strong agreement among expert respondents. ${ }^{5}$ Furthermore, a systematic review described a correlation between radiotherapy delay and oncologic outcomes, where a reduced loco-regional control was noted in aggressive head and neck tumors. ${ }^{6}$

Repeat simulation and planning is defined as an additional computed tomography (CT) scan done after an initial simulation had already been acquired, whether actual treatment had commenced or not. Although avoidable, certain circumstances may determine the need for this process which has inherent costs to resources, patient convenience, and treatment course. A study by Metzger evaluating frequencies for all cancer histologies found that the repeat simulation rate in 683 patients with head and neck cancer was relatively high at $3.07 \%$, attributed primarily to changes in anatomy from the delay of treatment initiation. ${ }^{7}$

The possible causes for repeat simulation include set up changes, immobilization devices, and changes in RT plan. Changes in the set up that typically lead to repeat simulation include radiotherapy field adjustments and the need for patient repositioning. This may be due to anatomic changes (weight gain/loss) and/or patient discomfort with the planned set up upon initiation of radiotherapy. ${ }^{8-9}$ Field adjustments may be required if there is any need for positional changes to account for delays in treatment initiation, such as the need to hyperextend the neck for oral cavity cancers. Meanwhile, radiotherapy plan changes may come from the discretion of the treating physician beyond the other aforementioned reasons, to determine the need for repeat treatment planning. Any attempt to distinguish between random occurrences and systemic processes is difficult since influences may be intertwined for both suspected as the culprit for repeat simulation.

On March 16, 2020, the island of Luzon was placed under Enhanced Community Quarantine as the Philippine government's precaution against the disease caused by the novel SARS-CoV-2 virus or COVID-19. The University of the Philippines-Philippine General Hospital was designated as a COVID-19 referral center, consequently halting regular operations of its radiotherapy facilities. During this period, only admitted patients with emergent conditions were catered, resulting in the postponement of its high-volume outpatient census and repeat simulations becoming noticeably rampant. Even with maximal efforts of this institution to minimize treatment delays, the real-world effects of pandemic quarantine measures on the logistics and compliance to daily radiotherapy remain significant. ${ }^{10}$

This study thus aims to compare the incidence of repeat radiotherapy planning, as a surrogate of tumor progression, among patients with head and neck cancer treated before and during the COVID-19 pandemic in an ambispective design. Various patient- and/or treatment-related factors, including treatment delay, that may associate with the likelihood of repeat simulation were also investigated in this review.

\section{MATERIALS AND METHODS}

\section{Patients}

All patients diagnosed with malignant neoplasms of the head and neck region who underwent CT-simulation for external beam radiation therapy from September 2019 to August 2020 (6 months before and after the start of pandemic) were included in this study. Patients with sole intracranial disease and those with treatment withheld or not planned to complete course of treatment were excluded.

\section{Study Procedure and Data Collection}

After obtaining institutional review board approval, records of patients satisfying the inclusion criteria were retrospectively reviewed, particularly those of patients diagnosed with cancer localized to the head and neck region having undergone initial CT simulation and to be treated or were undergoing external beam radiotherapy in the institution during the defined study period. The respective outpatient records, radiation therapy charts, CT-simulation images, and treatment plans of patients identified were accessed and reviewed.

Patients were classified into either one of two groups ambispectively; 1) a retrospective arm of patients who have completed radiation therapy prior to the start of pandemic (September 2019 to March 16, 2020), and 2) a prospective arm of patients who underwent CT simulation and scheduled or already undergoing radiation therapy during the pandemic (March 17 to August 30, 2020). 
A standard case record form was used for data recording. This contained the patient's demographic information, history of present illness, pre-treatment diagnosis and staging, and treatment-related parameters. The principal investigator assigned codes to the patients to assure confidentiality. Data gathered was reported as group data.

For this study, repeat simulation and planning were defined as an additional computed tomography simulation done after an initial simulation and radiotherapy treatment plan had already been made, whether actual treatment had commenced or not. In addition to the need for re-simulation, the following baseline information was tabulated from the patient's records: the patient's age, sex, tumor histology, tumor site, primary or metastatic, stage at time of treatment, radiation doses and use of chemotherapy. Other pertinent data were also included in data collection: intervals between initial CT simulation and treatment initiation, repeat simulation, and treatment continuation, as well as any treatment interruptions and length of delays mid-treatment. For patients in the arm undergoing radiotherapy during the pandemic, the need for repeat simulation was determined independently by the attending physician, with the outcome recorded upon initiation or resumption of the patient's radiotherapy course. Etiologies for repeat simulation for both arms were gathered and recorded. The records were followed during the course of treatment, and data was retrieved until the completion of radiotherapy.

Data extracted from the charts and other patient records were consolidated. Essential data were tabulated using Microsoft Excel.

\section{Statistical Methods and Data Analysis}

This study utilized a purposive sampling for an ambispective study design. A minimum sample of 72 was required for this study based on $3.07 \%$ prevalence of resimulation rate on head and neck, ${ }^{11} 5 \%$ level of significance and $4 \%$ desired precision. ${ }^{12}$

Descriptive statistics were used to summarize the demographic and clinical characteristics of the patients. Frequency and proportion were used for categorical variables, median and interquartile range for non-normally distributed continuous variables, and mean and SD for normally distributed continuous variables.

Independent sample T-test, Mann-Whitney U test, and Fisher's Exact/Chi-square test were used to determine the difference of mean, rank and frequency, respectively, between patients with initiating/undergoing radiotherapy during the pandemic versus patients whose radiotherapy done before the pandemic. Odds ratio and corresponding 95\% confidence intervals from binary logistic regression were computed to determine significant factors of repeat simulation as well as its subgroup analysis among patients with treatment interruptions. All statistical tests were twotailed tests. Shapiro-Wilk was used to test the normality of the continuous variables. Missing values were neither replaced nor estimated. Null hypotheses were rejected at $0.05 \alpha$-level of significance. STATA 13.1 was used for data analysis.

\section{RESULTS}

A total of 72 patients were included in the study with an average age of 45 years (range: 7-82). Males (51.4\%) and females (48.6\%) were equally represented. Among the subsites, involvement of the nasopharynx was most common (33.3\%) while the remainder (36.1\%) comprised of neck primaries and the paranasal sinuses. Three patients (4.2\%) had metastatic disease to the neck from a distant primary. The majority of cases had a squamous cell histology (61.1\%) and locally advanced, non-metastatic stage IV disease (59.7\%). Non-squamous histologies included adenoid cystic carcinomas, thyroid and cutaneous histologies, among others. Use of chemotherapy was concurrent in 32 patients (45.1\%) and was used in the induction setting in only 5 patients (7.04\%). Aside from tumor site, baseline characteristics were balanced between the pre-pandemic and pandemic groups as displayed in Table 1.

In terms of radiotherapy, the population had a mean of 32.5 ( $\mathrm{SD} \pm 17,239)$ fractions and number of dose fractions were not statistically different between the two groups $(\mathrm{p}=$ 0.425 ). Fractionation was mostly conventional (57 patients, 79.1\%) with significantly higher percentage of patients undergoing hypofractionation in the pre-pandemic group ( $76.9 \%$ vs $23.1 \%, p=0.029)$. Radiotherapy technique was $3 \mathrm{D}$-conformal techniques (72.2\%) for most patients in both groups.

Median time to radiotherapy initiation for the entire sample was 37 days ( $\mathrm{SD} \pm 37$, range of $0-278$ ) from initial CT simulation, and there was a paradoxical trend towards shorter average delays during the pandemic $(\mathrm{p}=0.774)$. As outlined in Table 2, there is a significant difference observed in the proportion of cases having repeat simulation done between the groups $(p=0.007)$, with a higher repeat simulation rate in the pandemic group ( $76.2 \%$ vs $23.8 \%)$. A non-significant increase in delay from initial to repeat simulation was observed in the pandemic group (170.5 vs 63 days, $\mathrm{p}=0.216)$. Incidence of treatment interruptions was higher in the pandemic group $(64.0 \%$ vs $36.0 \%, \mathrm{p}$ $=0.118$ ) though non-significant, with the interval from stoppage to resumption displaying a higher trend as well in this group ( 14.5 vs 6 days, $p=0.124)$. Overall treatment time was similar in the subset of patients undergoing repeat simulation in both groups ( 50 vs 61 days, $\mathrm{p}=0.87$ ) as well as in the remainder of patients with no repeat simulation done (48 vs 42.5 days, $\mathrm{p}=0.67$ ).

Changes in radiotherapy plan (13.9\%) were among the most common documented etiologies of repeat simulation, the causes of which comprised of prescription changes and addition or extension of radiotherapy fields. Other reasons included tumor growth, shrinkage, and set-up changes. 
Table 1. Baseline clinico-demographic characteristics of the study population

\begin{tabular}{|c|c|c|c|c|}
\hline \multirow{2}{*}{ Variable } & Total $(n=72)$ & Pre-pandemic ( $n=35)$ & Pandemic Period $(n=37)$ & \multirow{2}{*}{ p-value } \\
\hline & \multicolumn{3}{|c|}{ Frequency (\%); Mean \pm SD; Median (IQR) } & \\
\hline Age (years) & $45.46(16.6)$ & $46.57(15.4)$ & $44.41(17.8)$ & 0.584 \\
\hline Sex & & & & 0.155 \\
\hline Male & $37(51.4)$ & 21 & 16 & \\
\hline Female & $35(48.6)$ & 14 & 21 & \\
\hline Tumor Site & & & & 0.042 \\
\hline Nasopharynx & $24(33.3)$ & 10 & 14 & \\
\hline Oral Cavity & $12(16.7)$ & 4 & 8 & \\
\hline Oropharynx & $1(1.4)$ & 1 & - & \\
\hline Larynx & $4(5.6)$ & 2 & 2 & \\
\hline Other Pharynx & $2(2.8)$ & - & 2 & \\
\hline Other Neck Primary & $26(36.1)$ & 18 & 8 & \\
\hline Metastatic & $3(4.2)$ & 3 & - & \\
\hline Tumor Histology & & & & 0.248 \\
\hline SCCA & $44(61.1)$ & 19 & 25 & \\
\hline Other & $28(38.9)$ & 16 & 12 & \\
\hline Clinical TNM Stage at Time of Treatment & & & & 0.417 \\
\hline Benign & $7(9.7)$ & 4 & 3 & \\
\hline I & $1(1.4)$ & - & 1 & \\
\hline II & $6(8.3)$ & 3 & 3 & \\
\hline III & $15(20.8)$ & 10 & 5 & \\
\hline IV & $43(59.7)$ & 18 & 25 & \\
\hline Total Number of Dose Fractions & $32.5(17,2-39)$ & $30(25,5-35)$ & $33(10,2-39)$ & 0.425 \\
\hline Fractionation & & & & 0.029 \\
\hline Hyperfractionation & $2(2.8)$ & - & 2 & \\
\hline Conventional & $57(79.2)$ & 25 & 32 & \\
\hline Hypofractionation & $13(18.0)$ & 10 & & \\
\hline Planning Technique & & & & 0.295 \\
\hline $2 \mathrm{D}$ & $2(2.8)$ & 2 & - & \\
\hline $3 \mathrm{D}$ & $52(72.2)$ & 26 & 26 & \\
\hline IMRT & $18(25.0)$ & 7 & 11 & \\
\hline Use of chemotherapy & & & & 0.488 \\
\hline Induction & $5(7.0)$ & 2 & 3 & \\
\hline Concurrent & $32(45.1)$ & 13 & 19 & \\
\hline Sequential/Other & $7(9.9)$ & 4 & 3 & \\
\hline None & $27(38.0)$ & 16 & 11 & \\
\hline Interval from initial CT simulation to first RT fraction & $37(45,0-278)$ & $47(43,1-22)$ & $29(59,0-278)$ & 0.774 \\
\hline
\end{tabular}

Table 2. Incidence of repeat simulation, treatment delays/interruptions and related data

\begin{tabular}{|c|c|c|c|}
\hline \multirow{2}{*}{ Parameter } & Pre-pandemic $(n=35)$ & Pandemic Period $(n=37)$ & \multirow[t]{2}{*}{ p-value } \\
\hline & \multicolumn{2}{|c|}{ Frequency (\%) } & \\
\hline Interval from initial CT simulation to first RT fraction (days) & $47(43,1-22)$ & $29(59,0-278)$ & 0.7740 \\
\hline Repeat simulation rate & $5(23.8)$ & $16(76.2)$ & 0.0070 \\
\hline \multirow{2}{*}{ Parameter } & Pre-pandemic $(n=5)$ & Pandemic Period $(n=16)$ & \multirow{2}{*}{ p-value } \\
\hline & \multicolumn{2}{|c|}{ Mean \pm SD; Median (IQR) } & \\
\hline Interval from initial to repeat simulation (days) & $63(84,20-253)$ & $170.5(84.5,14-261)$ & 0.2160 \\
\hline Overall treatment time (days) & $61(81,25-221)$ & $50(97.5,3-329)$ & 0.6793 \\
\hline Overall treatment time for patients without repeat simulation (days) & $42.5(38,5-66)$ & $48(18,10-75)$ & 0.7302 \\
\hline Patients with treatment interruptions & $9(25.7)$ & $16(43.2)$ & SS \\
\hline $\begin{array}{l}\text { Interval from treatment stoppage to treatment continuation among those } \\
\text { with delay (days) }\end{array}$ & $6(36.5,2-207)$ & $14.5(73,4-303)$ & 0.1240 \\
\hline
\end{tabular}


Table 3. Incidence of etiologies of repeat simulation among respondents

\begin{tabular}{lcc} 
Reason for repeat simulation & $\begin{array}{c}\text { Rate of } \\
\text { occurrence (\%) }\end{array}$ & $\begin{array}{c}\text { Number of } \\
\text { patients ( } \mathbf{~ = ~ 7 2 ) ~}\end{array}$ \\
\hline Radiotherapy plan changes & 13.9 & 10 \\
Tumor growth & 6.9 & 5 \\
Tumor shrinkage & 5.6 & 4 \\
Set up changes & 2.8 & 2 \\
\hline
\end{tabular}

A summary of the number of patients affected and their corresponding percentages are displayed in Table 3.

On multivariate regression analysis and adjusting for all other factors as displayed in Table 4, pandemic grouping was significantly associated with repeat simulation, with participants treating in the pandemic period having higher odds of having a repeat simulation than those treated pre-pandemic $(\mathrm{p}=0.007$; odds ratio $(\mathrm{OR}) 7.61,95 \% \mathrm{CI}$ 1.75-33.20). The interval from initial CT simulation to RT initiation was also significantly associated with repeat simulation. Those who had an interval of more than 28 days had a higher incidence of repeat simulation $(\mathrm{p}=0.005$, OR 13.59; 95\% CI 2.2382.87). An interval from treatment stoppage to treatment continuation of more than 14 days also had a significantly higher odds of repeat simulation as compared to those patients experiencing no delays midtreatment ( $\mathrm{p}=0.004$, OR 23.21; 95\% CI 2.79-193.29). However, there is no sufficient evidence to conclude that those who had an interval from treatment stoppage to treatment continuation of fewer than 14 days had different odds of having a repeat simulation than those who have no delays $(\mathrm{p}=0.757$, OR 0.76 ; 95\% CI 0.13-4.43).

\section{DISCUSSION}

The need for repeat simulation and planning represents a deleterious effect of treatment delay, resulting in additional expense, radiation exposure, and further delay. Head and neck sites are commonplace for repeat CT simulations, largely due to changes in anatomy from the aggressive nature of such tumors. ${ }^{7}$ The necessity for repeat planning is paramount with more conformal therapies such as intensitymodulated radiotherapy (IMRT) becoming available, where it is deemed essential in ensuring adequate target doses and safe normal tissue exposure. ${ }^{9,13}$

In this study that included non-metastatic, locally advanced head and neck cancers, as well as primary cancers metastatic to this location, the median time to radiotherapy initiation was 37 days, with a range from zero to as much as 278 days. Such protraction is known to be detrimental to oncologic outcomes, given that this interval should ideally not exceed 40 days from diagnosis, to preserve tumor control and survival. ${ }^{14}$ Consequently, a significant association was observed between such interval with the likelihood of repeat simulation, as a surrogate outcome for tumor progression. Those who had an interval from initial CT simulation to first radiotherapy fraction of more than 28 days had 4.77 times higher odds of having a repeat simulation than those who had an interval shorter than a month. Those who had mid-treatment delays more than 14 days also notably had a significantly higher chance of repeat simulation by more than twenty-fold.

For head and neck cancers, locoregional recurrence rates and absolute survival are significantly associated with delay of treatment initiation. ${ }^{12,18}$ Radiobiologic models, as well as systematic reviews on clinical outcomes, have suggested that postponement of treatment caused by referral delays and increasing waiting times are associated with increased tumor doubling times and worse tumor control probability. ${ }^{15}$ A study evaluated the effect of waiting times on tumor progression among head and neck cancers and found that $62 \%$ of patients had a measurable increase in tumor volume with a median of $46 \%$ increase in a median duration of 28 days or 4 weeks. Another study noted a significant increase in the gross tumor volume of a median $16 \%$ over a median 13 days. ${ }^{16}$

Table 4. Association of Pandemic Period Group, Delayed Radiotherapy Initiation, and Interruptions with Likelihood of Repeat Simulation

\begin{tabular}{|c|c|c|c|c|c|c|}
\hline & \multicolumn{3}{|c|}{ Univariable } & \multicolumn{3}{|c|}{ Multivariable } \\
\hline & Odds Ratio & $95 \% \mathrm{Cl}$ & p-value & Odds Ratio & $95 \% \mathrm{Cl}$ & p-value \\
\hline \multicolumn{7}{|l|}{ Pandemic Group } \\
\hline Pre-pandemic & Ref. & & & Ref. & & \\
\hline Pandemic Period & 4.57 & {$[1.45,14.42]$} & 0.010 & 7.61 & {$[1.75,33.20]$} & 0.007 \\
\hline \multicolumn{7}{|c|}{ Interval from initial CT simulation to first RT } \\
\hline$<28$ days & Ref. & & & Ref. & & \\
\hline$\geq 28$ days & 5.77 & {$[1.51,22.03]$} & 0.010 & 13.59 & {$[2.23,82.87]$} & 0.005 \\
\hline \multicolumn{7}{|c|}{ Interval from treatment stoppage to treatment continuation } \\
\hline No delay & Ref. & & & Ref. & & \\
\hline Delay $<14$ days & 1.71 & {$[0.38,7.74]$} & 0.487 & 0.76 & {$[0.13,4.43]$} & 0.757 \\
\hline Delay $\geq 14$ days & 18.38 & {$[3.50,96.36]$} & 0.001 & 23.21 & {$[2.79,93.29]$} & 0.004 \\
\hline
\end{tabular}

95\% Cl - 95\% Confidence Interval; Ref. - Reference 
The sample studied was representative of the typical treatment census in this institution, comprising of a high proportion of nasopharyngeal cancers as well as occasional metastatic lesions from sites other than the head and neck. Unsurprisingly, a significantly higher incidence of repeat simulation and radiotherapy planning was observed in patients treated during the pandemic ( $76.2 \%$ vs $23.8 \%)$. Radiotherapy plan changes were the most common documented etiology of repeat simulation and this broad category consisted of prescription changes and addition or extension of radiotherapy fields. Other reasons included tumor and set up changes, although it can be surmised that tumor growth was a frequent occurrence given the nature of plan changes compensating such anatomic progression.

Quarantine measures during this pandemic have indeed adversely affected outcomes in this study population, with a significant six-fold probability of having a repeat simulation in the group treated during the pandemic. Furthermore, in this group of patients undergoing repeat simulation, a trend toward a longer interval to the start of radiotherapy after re-simulation was noted during the pandemic (170 days vs. 63 days). Overall treatment time among patients undergoing repeat simulation was just slightly lower in the pandemic group (50 vs 61 days) but was slightly higher in the same group for those with no repeat simulation (48 vs 42 days), although non-significant in both cases. This quantity representing delay is consequential to the likelihood of tumor progression, with a study defining overall treatment time prolongation as more than 56 days for definitive cases and more than 49 days for post-operative cases. ${ }^{17}$

In line with this, radiotherapy should be delivered at the earliest opportunity if the treatment is curative, given that there is as much as $16 \%$ increase risk of death for every month of delay of radiotherapy. ${ }^{13}$ Data is robust based on well-established guidelines that time is of the essence for head and neck cancers and that treatment protraction is to be avoided, even in this time of pandemic.

\section{CONCLUSION}

To conclude, our team conducted a retrospective analysis to determine the incidence of repeat radiotherapy simulation and planning in patients diagnosed with cancer localized to the head and neck region, as well as to describe patientrelated, radiotherapy-related, and/or other factors that may associate with such outcomes in this population during the COVID-19 pandemic.

This cohort of patients included those undergoing treatment up to six months into the pandemic and at this time, a higher incidence of repeat simulation was observed as compared to a similar group before the pandemic. Treatment interruptions were more common among those treated during the pandemic and along with delayed initiation, were both significantly associated with the likelihood for repeat simulation. With further backlogs encountered from a limited treatment census and restrictive logistics during this pandemic, strategies should be employed to prevent the occurrence of disease progression. Such is manifested with these outcomes in head and neck cancer patients, where there is a need to address the logistical and resource burden that arises with such unplanned, repeat radiotherapy planning.

\section{Recommendations}

The limited population sample restricts formulating conclusions on causality for the variables of delay that have been correlated with the incidence of repeat simulation. Given that the sample was computed to fulfill the primary objective of determining the repeat simulation rates and detecting a significant difference between the groups based on pandemic period, further inclusion of all head and neck cancer patients seen in the institution may rectify this limitation and allow for in-depth multivariate analysis.

\section{Statement of Authorship}

Both authors participated in the data collection and analysis and approved the final version submitted.

\section{Author Disclosure}

Both authors declared no conflicts of interest.

\section{Funding Source}

This study was self-funded.

\section{REFERENCES}

1. Brady LW, Wazer DE, Perez CA. Perez \& Brady's principles and practice of radiation oncology. Lippincott Williams \& Wilkins; 2013 May 6. pp. 859-60.

2. Philippine Cancer Society. 2015 Philippine Cancer Facts and Estimates [Internet]. 2015 [cited $2020 \mathrm{Apr}$ ]. Available from: http:// www.philcancer.org.ph/wp-content/uploads/2017/07/2015-PCS-CaFacts-Estimates_CAN090516.pdf

3. Ontario Health: Cancer Care Ontario, Pandemic Planning Clinical Guide for Patients with Cancer [Internet]. 2020 [cited 2020 Apr]. Available from: https://www.accc-cancer.org/docs/documents/ cancer-program-fundamentals/oh-cco-pandemic-planning-clinicalguideline_final_2020-03-10.pdf?sfvrsn=d2f04347_2

4. Withers HR, Taylor JM, Maciejewski B. The hazard of accelerated tumor clonogen repopulation during radiotherapy. Acta Oncol. 1988 Jan 1; 27(2):131-46.

5. American Society for Radiation Oncology, COVID-19 Recommendations to Radiation Oncology Clinical Practices [Internet]. 2020 [cited $2020 \mathrm{Apr}$ ]. Available from: https://www.astro. org/Daily-Practice/COVID-19-Recommendations-and-Information/ COVID-19-FAQs

6. Huang J, Barbera L, Brouwers M, Browman G, Mackillop WJ. Does delay in starting treatment affect the outcomes of radiotherapy? A systematic review. J Clin Oncol. 2003 Feb 1; 21(3):555-63.

7. Metzger A, Renz P, Hasan S, Karlovits S, Sohn J, Gresswell S. Unforeseen computed tomography resimulation for initial radiation planning: associated factors and clinical impact. Adv Radiat Oncol. 2019 Oct 1; 4(4):716-21.

8. Hansen EK, Bucci MK, Quivey JM, Weinberg V, Xia P. Repeat CT imaging and replanning during the course of IMRT for head-andneck cancer. Int J Radiat Oncol Biol Phys. 2006 Feb 1; 64(2):355-62. 
9. Gros SA, Xu W, Roeske JC, Choi M, Emami B, Surucu M. A novel surrogate to identify anatomical changes during radiotherapy of head and neck cancer patients. Med Phys. 2017 Mar; 44(3):924-34

10. Chen YL, Hsu FM, Tsai CJ, Cheng JC. Efforts to reduce the impact of Coronavirus diseases 2019 outbreak on radiation oncology in Taiwan. Adv Radiat Oncol. 2020; 5(4):534-7.

11. Thomson DJ, Palma D, Guckenberger M, Balermpas P, Beitler JJ, Blanchard $\mathrm{P}$, et al. Practice recommendations for risk-adapted head and neck cancer radiation therapy during the COVID-19 pandemic: An ASTRO-ESTRO consensus statement. Int J Radiat Oncol Biol Phys. 2020 Jul 15; 107(4):618-27.

12. Peacock JL, Peacock PJ. Research design. In: Peacock JL, Peacock PJ. Oxford handbook of Medical Statistics. United States: Oxford University Press; 2011. pp. 60-61.

13. Kishan AU, Cui J, Wang PC, Daly ME, Purdy JA, Chen AM. Quantification of gross tumour volume changes between simulation and first day of radiotherapy for patients with locally advanced malignancies of the lung and head/neck. J Med Imaging Radiat Oncol. 2014 Oct; 58(5):618-24.
14. Fortin A, Bairati I, Albert M, Moore L, Allard J, Couture C. Effect of treatment delay on outcome of patients with early-stage head-andneck carcinoma receiving radical radiotherapy. Int J Radiat Oncol Biol Phys. 2002 Mar 15; 52(4):929-36.

15. Wyatt RM, Beddoe AH, Dale RG. The effects of delays in radiotherapy treatment on tumour control. Phys Med Biol. 2002 Dec 23; 48(2):139.

16. Barker Jr JL, Garden AS, Ang KK, O'Daniel JC, Wang H, Court LE, et al. Quantification of volumetric and geometric changes occurring during fractionated radiotherapy for head-and-neck cancer using an integrated CT/linear accelerator system. Int J Radiat Oncol Biol Phys. 2004 Jul 15; 59(4):960-70.

17. Hansen O, Overgaard J, Hansen HS, Overgaard M, Høyer M, Jørgensen $\mathrm{KE}$, et al. Importance of overall treatment time for the outcome of radiotherapy of advanced head and neck carcinoma: dependency on tumor differentiation. Radiother Oncol. 1997 Apr 1; 43(1):47-51.

\title{
Have you read the current trends in
} Medical and Health Research in the Philippines?

\section{Acta Medica Philippina The National Health Science Journal}

\author{
Access Online: www.actamedicaphilippina.upm.edu.ph
}

